\section{Seeing more clearly}

Microscope and XRD enhancements

\section{wuw.jeol.com}

High-resolution SEM

The JSM-7400F SEM has been designed specifically for imaging at the nanometre scale. It is claimed to deliver $50 \%$ higher resolution than current models, attaining $1.5 \mathrm{~nm}$ at $1 \mathrm{kV}$. The advanced lens design includes an SE enhancer, an $r$-filter and an in-lens back scatterer to enhance image quality, reduce charging contrast and heighten $z$ contrast. The large chamber allows most samples to be analysed without altering them. The load locks and specimen stages can handle samples up to

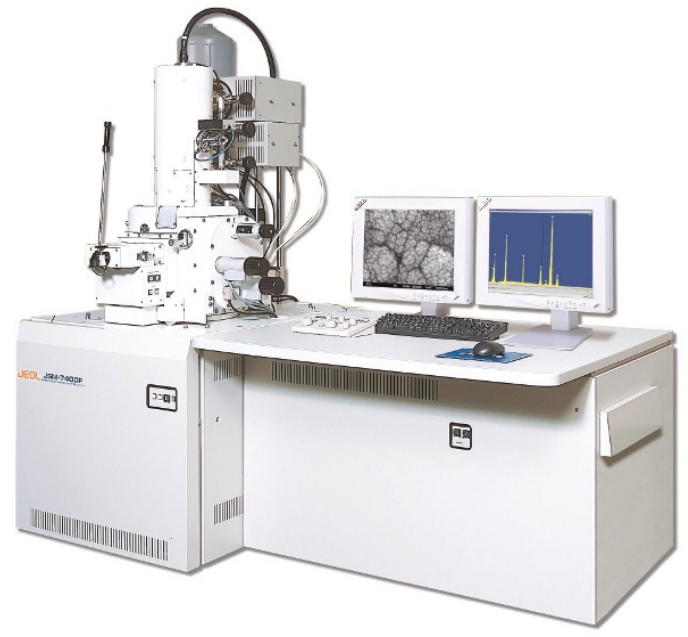

$200 \mathrm{~mm}$ in diameter for analysis over transverse ranges up to $140 \times 80 \mathrm{~mm}$. Analytical geometry enables a working distance of $8 \mathrm{~mm}$ at a takeoff angle of $35^{\circ}$. An EBSD detector can be mounted and used simultaneously with the EDS.

NANO-VIEW, NANO-LP, NANO-PDQ50 Mad City Labs

Nanopositioning systems for microscopes

Mad City Labs offer three new positioning systems with nanometre precision for microscopy
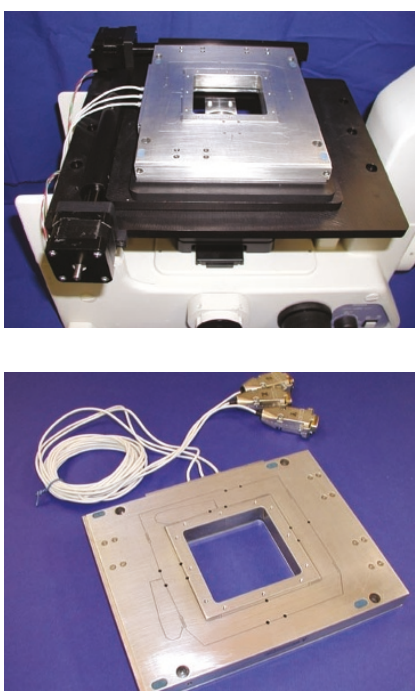

applications. The systems feature piezoelectric sensors for absolute position measurement and subnanometre accuracy when operated under closed-loop control with the Nano-Drive.

The Nano-View system is designed to be integrated with inverted optical microscopes for applications such as single-molecule spectroscopy. The nanopositioning stage with integrated encoders is designed to allow accurate position tracking; it has up to $4 \mathrm{~cm}$ of travel with $20 \mathrm{~nm}$ resolution. The Nano-LP series is a range of ultralow profile, three-axis nanopositioning systems, which offer up to $200 \times 200 \times 100 \mu \mathrm{m}$ of travel

\section{WWW.madcitylabs.com}

with 1-nm resolution. They are intended to be easily integrated into existing instrumentation, and offer absolute imaging of cells and other nanostructures. The Nano-PDQ50 is a two-axis system offering a compact footprint with a large centre aperture, while still providing fast performance with a resonant frequency of greater than $1 \mathrm{kHz}$.

The setup involves parallel uncoupled motion, providing $50 \mu \mathrm{m}$ of travel in both $x$ and $y$ directions, and is ideal for applications requiring high scanning rates or large-load capacity. It is particularly suitable when bleaching is performed and for the calibration of optical traps.

South Bay Technology $\quad$ www.southbaytech.com

Ultra-thin films enhance EM imaging

The IBS/e is a new tabletop thin-film deposition and etching system. It is designed to improve high-resolution electron microscopy imaging by depositing ultra-thin, fine-grain metal and carbide films on to contamination-free surfaces that have been etched to reveal fine structures. The sputtered material evolves controllably and repeatedly with an energy of $25 \mathrm{eV}$. There are no heat or radiation artefacts to spoil specimen detail.

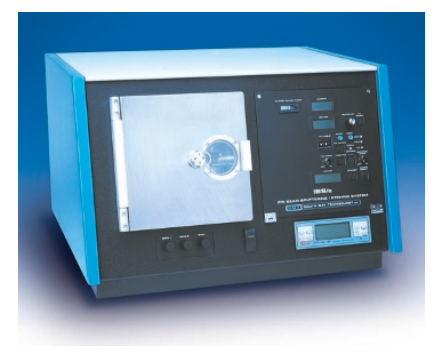

The dual-axis motion of the stage ensures uniform coverage of cracks and crevices in specimens up to $50 \mathrm{~mm}$ in diameter. Properly deposited films are said to be beyond the resolving power of the highest magnification of field-emission scanning electron microscopy.

\section{X'PERT EPITAXY

Philips Analytical $\quad$ WWw.analytical.philips.com

Rocking curves made easy

Philips Analytical have enhanced their X'Pert Epitaxy XRD software to include automatic fitting of simulated rocking curves to measured scans, and support for the new data format based on the industry standard, XML. The software can be used on all single-crystal and highly textured thinlayer samples, such as semiconductor, superconductor and magnetic layers.

The software is intended for visualizing and analysing single and twoaxis scans recorded on the X'Pert PRO MRD and high-resolution

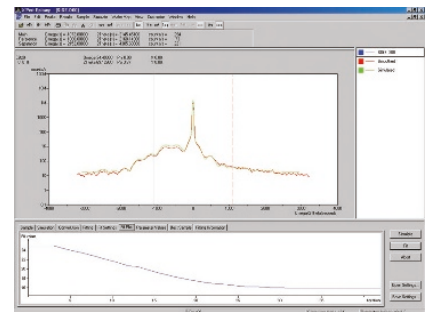

diffractometers. Three packages are available: X’Pert Epitaxy Graphics supports extensive analysis of rocking curves and two-axis scans of cubic and hexagonal semiconductor structures; X'Pert Epitaxy adds a facility for simulation of rocking curves; and the X'Pert Smoothfit adds automatic fitting for rocking curves. 